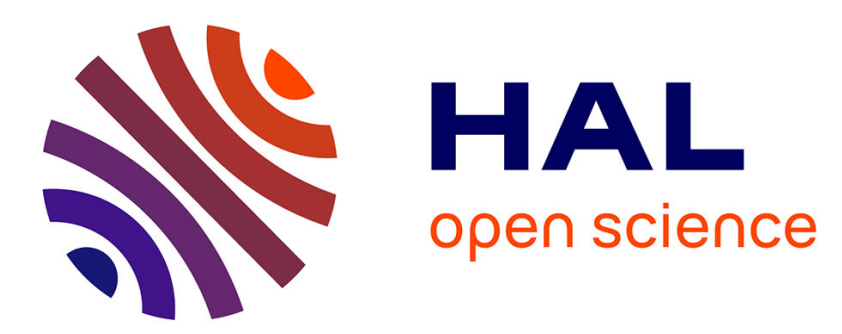

\title{
An opto-electronic system for in-situ determination of peroxide value and total phenol content in olive oil
} Marco Grossi, Giuseppe Di Lecce, Marco Arru, Tullia Gallina Toschi, Bruno Riccò

\section{- To cite this version:}

Marco Grossi, Giuseppe Di Lecce, Marco Arru, Tullia Gallina Toschi, Bruno Riccò. An opto-electronic system for in-situ determination of peroxide value and total phenol content in olive oil. Journal of Food Engineering, 2015, 146, pp.1-7. 10.1016/j.jfoodeng.2014.08.015 hal-01276359

\section{HAL Id: hal-01276359 \\ https://hal.science/hal-01276359}

Submitted on 25 Feb 2016

HAL is a multi-disciplinary open access archive for the deposit and dissemination of scientific research documents, whether they are published or not. The documents may come from teaching and research institutions in France or abroad, or from public or private research centers.
L'archive ouverte pluridisciplinaire HAL, est destinée au dépôt et à la diffusion de documents scientifiques de niveau recherche, publiés ou non, émanant des établissements d'enseignement et de recherche français ou étrangers, des laboratoires publics ou privés. 
1 An opto-electronic system for in-situ determination of peroxide value and total phenol content

2 in olive oil

4 Marco Grossi $^{\mathrm{a},{ }^{*},}$, Giuseppe Di Lecce ${ }^{\mathrm{b}}$, Marco Arru ${ }^{\mathrm{a}}$, Tullia Gallina Toschi ${ }^{\mathrm{c}}$, Bruno Riccò $^{\mathrm{a}}$

* Corresponding author : marco.grossi8@unibo.it, Tel. 0039-0512093082, Fax 0039-0512093785

6 a Department of Electrical Energy and Information Engineering "Guglielmo Marconi” (DEI),

7 University of Bologna, Bologna, Italy

8 b Department of Food Chemistry, Eurofins Chemical Control, Cuneo, Italy

9 c Department of Agricultural and Food Sciences - DISTAL, University of Bologna, Cesena, Italy

11 Abstract

12 The quality of olive oil is essentially determined by the product free acidity and peroxide value, 13 while the total phenol content is also important for a high antioxidant capacity. Generally, these 14 parameters are measured with laboratory analysis, that are expensive and may require a few days. 15 Thus, a cheap and easy technique usable by untrained personnel, "on-site" and producing results "in 16 real time" during production is desirable, particularly as far as small olive oil mills and packaging 17 centers are concerned. This paper describes a technique to determine peroxide value and total 18 phenol content in olive oil, that is based on the measurement of optical density of an emulsion 19 between a suitable chemical reagent and a small quantity of the oil of interest. The optical density is measured by illuminating the sample with a LED with peak wavelength of $569 \mathrm{~nm}$ for peroxide

21 value and $835 \mathrm{~nm}$ for total phenol content. The experimental results show good correlation $\left(\mathrm{R}^{2}=\right.$ 220.883 and 0.895 for peroxide value and total phenol content, respectively) between data measured 23 with the standard methodology and the technique of this work, implemented also in the form of a 24 portable embedded system.

Keywords: olive oil, peroxide value, total phenol content, sensor, optical density. 


\section{Introduction}

29 Olive oil is a vegetable lipid obtained by extraction process from olives (the fruits of Olea europaea

30 L., family Oleaceae) highly appreciated for its beneficial effects on human health, mainly due to a 31 high content of oleic acid and phenolic compounds (Tulipani et al., 2012). Clinical studies provide 32 evidence that regular olive oil consumption reduces the risk of coronary heart diseases (Keys et al., 33 1986), oxidative damage to DNA and RNA (Machowetz et al., 2007) and Alzheimer disease 34 (Abuznait et al., 2013; Monti et al., 2011).

35 Olive oil quality is related to its chemical composition, oxidative stability and sensory characteristics. Quality parameters, such as free acidity, peroxide value, UV extinction coefficients, fruity attribute, other sensory characteristics and defects, are strongly dependent on olives' ripeness

38 (Rotondi et al., 2004) and processing technology in the olive mills (Boselli et al., 2009). In addition, 39 the peroxide value, defined as milliequivalent of active oxygen per kilogram of oil (meq $\mathrm{O}_{2} / \mathrm{kg}$ oil) 40 and qualifying the oil primary oxidation, is also related to storage conditions (oxygen, light 41 exposure and temperature) after production. Another important quality parameter is the amount of phenolic compounds that contribute to the oil sensory taste producing a distinctive bitter and a 43 pungent perception (Gutierrez-Rosales et al., 2003). Phenolic compounds found in olive oil are principally secoiridoids (oleuropein and ligstroside isomers) and their derivatives, such as tyrosol and hydroxytyrosol, that exhibit a strong antioxidant activity: they act as free radicals traps protecting from heart disease and displaying anticancer activity (Notarnicola et al., 2011; Zanoni, 2014). Phenolic compounds are also largely responsible for the shelf-life of the oil (Lerma-Garcia et al., 2009).

49 The European Commission regulation No. 2568/91 and subsequent amendments define manual titration methods to measure acidity and peroxide value in olive oil (EEC 2568, 1991), to be carried

51 out in a laboratory environment by trained personnel. Instead, no official determination is currently 52 established for the total phenol content, usually determined using spectrophotometry or high 
performance liquid chromatography (HPLC), techniques requiring expensive instrumentation, a laboratory environment (IOC/T.20/Doc No 29, 2009; Tasioula-Margari and Okogeri, 2001) as well as preventive extraction of the polyphenols.

From the production point of view, the need to ship oil samples to a laboratory for analysis leads to high costs and long delays. Therefore, simple and fast techniques useable for on-site quality control are desirable, in particular for small oil mills and packaging centers. For this reason, innovative solutions have been proposed, such as: Near-InfraRed (NIR) spectroscopy (Armenta et al., 2007; Ozdemir and Ozturk, 2007) to estimate acidity and peroxide value; Time Domain Reflectometry (TDR) to determine water content (Ragni et al., 2012) and detect adulteration (Cataldo et al., 2012) in extra virgin olive oil; Rapid Fourier Transformed Infrared (FTIR) spectroscopy (Cerretani et al., 2010) and voltammetric sensors (Rodriguez-Mendez et al., 2008) to estimate total phenol content. However, all these techniques require expensive instrumentation and/or need frequent calibration for olives of different varieties, country of origin and harvest season.

As viable alternatives, amperometric and $\mathrm{pH}$-metric techniques have been proposed to measure peroxide value (Kardash-Strochkova et al., 2001; Adhoum and Monser, 2008) and total phenol content (Capannesi et al., 2000), but these methods are still at research stage and have been validated only on small amounts of samples in laboratory environment. Moreover, some techniques use toxic compounds (such as chloroform) to increase oil solubility in reagents, unsuitable for use in normal working environment.

Recently, we have proposed a novel technique based on Electrical Impedance Spectroscopy to measure olive oil acidity that is fast (response time in about 30 seconds) and can be easily implemented in the form of a low-cost portable embedded system (Grossi et al., 2013).

To complete this work, we here present a simple and effective technique to measure peroxide value and total phenol content in olive oil that, as will be shown, is fast, accurate and can be implemented in the form of a low-cost embedded electronic system. 


\section{Materials and methods}

80

81

82

\subsection{Technique}

The technique used in this work is based on the creation of an aqueous emulsion between the oil sample and a chemical reagent. The optical density (OD) of such an emulsion is determined by illuminating the sample with a LED and measuring the transmitted light through the sample with a photodiode. A large set of experimental results show a good correlation between the measured OD and the quality parameters determined by reference methods. The proposed technique is suitable to be implemented in the form of a portable instrument suitable for quick in-situ quality control, as will be discussed in sub-Section 3.3.

\subsection{Experimental set-up}

In order to validate the technique used in this work, measurements on olive oil samples have been initially carried out using an ad-hoc experimental set-up of bench-top instruments.

The sensor, depicted in Fig. 1 (a), consists of a cylindrical chamber (designed using Solid Edge by Siemens Systems and fabricated with a MakerBot Replicator 3D printer) devoted to host the $25 \mathrm{ml}$ polystyrene vial containing the emulsion between a suitable aqueous reagent (discussed in section 2.2) and the oil sample. The chamber features two diametrically opposed structures hosting a LED, used as light source and a photodiode to detect the light transmitted through the sample. In the case of peroxide value, the LED has a peak emission at $569 \mathrm{~nm}$ wavelength (biased with a $30 \mathrm{~mA}$ current), while the photodiode is a BPW21R by Vishai (with wavelength peak sensitivity at 565 $\mathrm{nm}$ ). In the case of total phenol content, instead, the LED has a peak emission at $835 \mathrm{~nm}$ (biased with a $80 \mathrm{~mA}$ current) and the photodiode is a OSD5-5T device by Centronic, with wavelength peak sensitivity between 700 and $900 \mathrm{~nm}$. As discussed in Section3, both the LED peak wavelengths have been chosen by means of preliminary measurements on phenolic and peroxide compounds using a SmartSpec 3000 spectrophotometer.

The experimental set-up is presented in Fig 1 (b). A DC power supply Agilent E3631A is used to provide the LED operating current $\left(\mathrm{I}_{\mathrm{LED}}\right)$ and the power supply for the operational amplifier. The 
105 photodiode current $\left(\mathrm{I}_{\text {photo }}\right)$, related to the detected light intensity, is converted into a voltage $\left(\mathrm{V}_{\text {out }}\right)$ 106 by a current-to-voltage converter. The voltage $\mathrm{V}_{\text {out }}$ is acquired by a NI USB-6211 Data Acquisition 107 (DAQ) board by National Instruments and transmitted to a PC for further analysis. All the software 108 for DAQ control, analysis, data presentation and filing has been realized with LabVIEW (National 109 Instruments). Statistical analysis on the experimental data has been carried out with Microsoft 110 EXCEL.

\subsection{Chemicals and media}

112 Phenolic reference standards (oleuropein, tyrosol, hydroxytyrosol, $p$-coumaric acid) and peroxide 113 compounds (hydrogen peroxide, $\mathrm{H}_{2} \mathrm{O}_{2}$, and tert-butyl hydroperoxide, $\mathrm{tBuOOH}$ ) were purchased 114 from Sigma-Aldrich (St. Louis, MO, USA). The reagent for peroxide value determination was

115 prepared diluting $8 \mathrm{~mL}$ of ferrous ion oxidation xylenol orange (FOX) reagent (an aqueous solution 116 of ferrous ammonium sulphate, sorbitol, sulphuric acid and xylenol orange, Sigma-Aldrich) 117 (Cheeseman, 2006) in $7 \mathrm{~mL}$ of distilled water. The reagent detects the peroxides concentration by 118 oxidation of ferrous ions $\mathrm{Fe}^{2+}$ to $\mathrm{Fe}^{3+}$ according to the following reaction:

$\mathrm{Fe}^{2+}+\mathrm{ROOH} \rightarrow \mathrm{Fe}^{3+}+\mathrm{RO}+\mathrm{OH}^{-}$

$120 \mathrm{Fe}^{3+}$ ions formed in the reaction are then detected using the dye xylenol orange which binds $\mathrm{Fe}^{3+}$ 121 forming a complex that strongly absorbs in the wavelength range $540-580 \mathrm{~nm}$.

122 For the total phenol content, instead, the reagent was prepared mixing: $13 \mathrm{~mL}$ of distilled water, 1 $123 \mathrm{~mL}$ of Folin-Ciocalteu reagent (a mixture of phosphomolybdate acid $\mathrm{H}_{3} \mathrm{PMo}_{12} \mathrm{O}_{40}$ and 124 phosphotungstate $\left.\mathrm{H}_{3} \mathrm{PW}_{12} \mathrm{O}_{40}\right)$ and $1 \mathrm{~mL}$ of sodium carbonate $\left(\mathrm{Na}_{2} \mathrm{CO}_{3}\right) 15 \%$ (i.e. $15 \mathrm{~g}$ di sodium 125 carbonate in $100 \mathrm{~mL}$ of distilled water). As a consequence of the reaction with the phenolic 126 compounds, the acids are reduced to tungsten and molybdenum oxides $\left(\mathrm{W}_{8} \mathrm{O}_{23}\right.$ and $\left.\mathrm{Mo}_{8} \mathrm{O}_{23}\right)$ 127 featuring a typical blue colour.

128 In both cases, the reagent was then mixed with $0.5 \mathrm{~mL}$ of the oil sample, all stirred for 30 seconds

129 to create the emulsion, then the vial is placed in the sensor for the measure. 
130 All the chemicals used in the experiments are of analytical grade. The olive oil samples used in the

131 experiments were purchased by local markets as well as olive oil mills.

$132 \quad 2.4$ Reference methods

133 Olive oil peroxide value has been determined by European standard reference method with starch as 134 indicator and sodium thiosulphate $\left(\mathrm{Na}_{2} \mathrm{~S}_{2} \mathrm{O}_{3}\right)$ as titrant, while total phenol content has been 135 determined according to spectrophotometric method proposed by Singleton and Rossi (Singleton 136 and Rossi, 1965). Phenolic fraction has been extracted using about $4 \mathrm{~g}$ of virgin olive oil (VOO) 137 with $5 \mathrm{~mL}$ of methanol:water (60:40). The extraction procedure has been repeated two time and the 138 hydroalcoholic fractions have been combined and evaporated by rotavapor up to complete dryness. 139 The concentrated extract has been dissolved in $5 \mathrm{~mL}$ of aqueous methanol (50\%), and filtered 140 through Minisart RC15 $(0.2 \mu \mathrm{m})$ regenerated cellulose syringe filters (Sartorius AG, Göttingen, D).

141 Total phenol content has been determined using the Folin-Ciocalteau reagent (Sigma-Aldrich, St. 142 Louis, MO, USA) and measuring at $750 \mathrm{~nm}$ with a Shimadzu spectrophotometer UV-VIS 1204 143 (Kyoto, Japan). The results have been expressed as mg of gallic acid per kg of VOO (gallic acid 144 calibration curve $\left.\mathrm{R}^{2}=0.993\right)$.

\section{Results and discussion}

147 The reagents response was initially characterized with peroxide and phenolic compounds using a 148 SmartSpec 3000 spectrophotometer. Then a set of 25 olive oil samples have been analyzed with the 149 technique and the bench-top set-up described in Section 2.1. Finally an electronic board has been 150 designed and fabricated to avoid the use of all bench-top instrumentation, thus demonstrating the 151 feasibility of a simple and economical instrument for easy, fast and in-situ analysis of olive oil.

\section{3.1. Spectral characterization of reagents response}

153 At first, the reagents were inoculated with known concentrations of peroxide and phenolic 154 compounds and the absorption spectra acquired with a SmartSpec 3000 spectrophotometer. 
In the case of phenols, four different compounds were tested: three of these are typically present at

156 high concentration (oleuropein, hydroxytyrosol and tyrosol) while the forth one ( $p$-coumaric acid) is present in small concentration. Fig. 2 (a) shows the absorption spectra in the case of different concentrations of oleuropein in the $400-800 \mathrm{~nm}$ wavelength range. As can be seen the different concentrations of phenolic compound can be discriminated for wavelength $>500 \mathrm{~nm}$ with the sensitivity increasing with the wavelength. In Fig. 2 (b) the absorbance measured at the wavelength of $800 \mathrm{~nm}$ is plotted vs. the phenol content for all the tested compounds. Except for tyrosol for which a higher sensitivity for concentrations below than $15 \mathrm{ppm}$ is registred, in all cases a linear relation between absorbance and concentration is found. The linear regression lines of the four cases are calculated to estimate the compound sensitivity (Abs800/ppm) to the reagent with the following results: 0.0199 (oleuropein), 0.1159 (hydroxytyrosol), 0.0248 ( $p$-coumaric acid), while in the case of tyrosol the sensitivity is 0.0755 for concentrations lower than $15 \mathrm{ppm}$ and 0.019843 for concentrations higher than 15 ppm. As depicted in Fig 2 (b), the intensity of blue color obtained by reduction of tungsten and molybdenum oxides is proportional to the concentration of each phenol but shows a different response respect to the phenol structure which reacted. In fact Folin-Ciocalteu detects the total reducing capacity that is different for different compounds (Apak et al., 2007). As 171 described in several paper, during the storage of VOO the trend of phenolic fraction, measured by 172 Folin-Ciocalteu assay showed a fluctuation due to the oxidation and lysis of phenolic compounds disperse in the matrix. This result confirms the different response factor that the reaction mixture presents towards individual phenols (Boselli et al., 2009; Fiori et al., 2014). In the case of peroxides, two different compounds were tested: hydrogen peroxide $\left(\mathrm{H}_{2} \mathrm{O}_{2}\right)$ and tertbutyl hydroperoxide (tBuOOH). The absorbance spectra in the wavelength range $400-800 \mathrm{~nm}$ are 177 shown in Fig. 3 (a) for different $\mathrm{tBuOOH}$ concentrations. As expected, the reaction of $\mathrm{Fe}^{2+}$ ions 178 with peroxide compounds results in the formation of $\mathrm{Fe}^{3+}$ ions that bind to xylenol orange 179 producing an absorbance peak between 560 and $580 \mathrm{~nm}$. The measured absorbance at $580 \mathrm{~nm}$ is 180 plotted vs. compound concentration for both $\mathrm{H}_{2} \mathrm{O}_{2}$ and $\mathrm{tBuOOH}$ in Fig. 3 (b). From the measured 
181 data, the absorbance can be empirically modelled as a linear function of the square root of the 182 compound concentration. The results indicate that $\mathrm{H}_{2} \mathrm{O}_{2}(y=0.297 \sqrt{x}+0.436)$ is characterized by 183 higher sensitivity than $\mathrm{tBuOOH}(\mathrm{y}=0.115 \sqrt{ } \mathrm{x}+0.265)$. In both cases a determination coefficient $\mathrm{R}^{2}$ 184 higher than 0.96 is achieved. The different response of FOX reagent to $\mathrm{H}_{2} \mathrm{O}_{2}$ and tBuOOH can be 185 due to the ability of sorbitol to scavenge hydroxyl radicals to yield peroxyl radicals which would 186 propagate $\mathrm{Fe}^{2+}$ oxidation (Jiang et al., 1990).

188 A set of 25 olive oil samples have been tested using the technique and the experimental set-up described in Section 2. The voltage $\mathrm{V}_{\text {out }}$ is acquired at time intervals of 5 seconds for a total of 1200 seconds for both tests (total phenol content and peroxide value).

191 In the case of total phenol content, the logarithm of $\mathrm{V}_{\text {out }}$ has been found to be linearly related with 192 the phenols concentration. The coefficient of determination $\mathrm{R}^{2}$ has been calculated after different time intervals and plotted as function of time in Fig. 4 (a): the correlation between $\log _{10}\left(\mathrm{~V}_{\text {out }} / \mathrm{V}_{\mathrm{M}}\right)$ (where $\mathrm{V}_{\mathrm{M}}$ is the measured voltage in the absence of oil sample, i.e. due to the reagent only) and the total phenol content increases with time, reaching a plateu after 600 seconds. Fig. 4 (b) shows the measured values of $\log _{10}\left(\mathrm{~V}_{\text {out }} / \mathrm{V}_{\mathrm{M}}\right)$ after 600 seconds plotted vs total phenol content as determined using the reference method. The linear regression line equation is $\mathrm{Y}=0.0343-2.389 \cdot 10^{-3} \cdot \mathrm{X}$ and the coefficient of determination 0.895 . The accuracy is slightly higher to what reported by Cerretani (Cerretani et al., 2010) when a $\mathrm{R}^{2}$ of 0.87 was obtained by FTIR attenuated total reflectance spectroscopy using a wavelength range $3610-816 \mathrm{~cm}^{-1}$ and a PLS chemometric analysis. The use 201 of an array of voltammetric sensors (Rodriguez-Mendez et al., 2008) reported an higher accuracy $202\left(\mathrm{R}^{2}=0.986\right)$, but the samples tested were only 6 olive oils and all above the $400 \mathrm{ppm}$; instead, in the present work, 25 samples were analysed and the level of detection achieved was of $100 \mathrm{ppm}$. In the case of peroxide value, a linear relation between the measured values of $V_{\text {out }} / \mathrm{V}_{\mathrm{M}}$ and the peroxide value has been found. As shown in Fig. 4 (c), the coefficient $\mathrm{R}^{2}$ increases with time reaching a plateau after about 600 seconds . Fig. 4 (d) shows the measured values of $\mathrm{V}_{\text {out }} / \mathrm{V}_{\mathrm{M}}$ plotted 
vs. the peroxide value as determined by the reference method. The linear regression line equation is

$208 \mathrm{Y}=0.6368-13.8 \cdot 10^{-3} \cdot \mathrm{X}$ and the coefficient of determination 0.883 . The dispersion between 209 measured data and the reference technique can be mainly associated with the property of the FOX 210 reagent that is able to determine all kinds of conjugated dienes including those without a peroxide 211 group, and not able to react with peroxides included in molecules without conjugated double bonds.

212 Furthermore the accuracy of the quantification can be also diminished by the presence of several 213 other compounds present in olive oil, such as carotenoids and chlorophyll, absorbing at 500-600 $214 \mathrm{~nm}$, and chain-breaking antioxidant able to reduce the color yield (Bou et al 2008). The obtained 215 accuracy is however higher than that reported in (Armenta et al., 2007) where a coefficient of 216 determination $\mathrm{R}^{2}$ of 0.6558 resulted by NIR spectroscopy combined with PLS chemometric 217 analysis.

218 In both cases there is a linear relation between the parameter measured with the experimental set-up $219\left(\log _{10}\left(\mathrm{~V}_{\text {out }} / \mathrm{V}_{\mathrm{M}}\right)\right.$ for total phenol content and $\mathrm{V}_{\text {out }} / \mathrm{V}_{\mathrm{M}}$ for peroxide value $)$ and the oil quality parameter measured with the reference methods. Thus, using the equation of the linear regression

221 line, the oil quality parameters can be estimated.

\subsection{Implementation as a portable embedded system}

223 To demonstrate that the technique proposed in this work can lead to a portable embedded system suitable for in-situ measurements, all the operations performed by the bench-top instrumentation and DAQ PC board have been implemented inside an electronic board designed "ad hoc", based on the $\mu$ controller Dspic33ep512mu810, whose schematic is presented in Fig. 5 (a). The LED is supplied with a square wave current (frequency $1 \mathrm{kHz}$ ) to allow removing the contribution of the environment radiation. In particular, a square wave voltage ranging from 0 to $830 \mathrm{mV}$ is generated by the $\mu$ controller and fed to the noninverting input of an operational amplifier. In turn, this latter drives a BJT transistor providing the LED with the supply current $\mathrm{I}_{\mathrm{LED}}$

231 (selectable with the value of the resistance $\mathrm{R}_{\mathrm{LED}}$ ). The radiation transmitted through the sample is 232 received by the photodiode that generates the current $\mathrm{I}_{\text {photo }}$ proportional to the incident radiation. An 
233 I/V converter generates a voltage $V_{\text {A }}$ proportional to $I_{\text {photo }}$. A couple of programmable switches 234 allows to select two different feedback resistors $\left(\mathrm{R}_{\mathrm{F} 1}\right.$ or $\left.\mathrm{R}_{\mathrm{F} 2}\right)$. The voltage $\mathrm{V}_{\mathrm{A}}$ is then fed to a $\mathrm{AC}$ 235 coupling stage that generates a square wave voltage $\left(V_{B}\right)$ with mean value $2.5 \mathrm{~V}$. Such a voltage is 236 then fed to a synchronous rectifier (built with an AD8271 difference amplifier and an ADG733 237 triple single pole double throw SPDT switch) that provides a $\mathrm{DC}$ voltage $\left(\mathrm{V}_{\mathrm{C}}\right)$ equal to the high 238 level of $\mathrm{V}_{\mathrm{B}}$. Then, a 18 bit ADC driver ADA4941 generates a differential voltage $\mathrm{V}_{\text {OuT }}$ 239 (proportional to $\mathrm{V}_{\mathrm{C}}$ ) that is fed to a 12 bit ADC (AD9220AR) providing the 12 bit digital 240 codification of $\mathrm{V}_{\text {OUT }}\left(\mathrm{D}_{\text {OUT }}\right)$ to be processed by the $\mu$ controller. The measured data are sent to a 241 portable PC (via USB interface) to display the results and file the data. The system has been built by 242 using low-cost electronics and its total cost has been estimated in about 300 \$. All the software has 243 been developed using LabVIEW. Fig. 5 (b) and (c) present pictures of the sensor, while Fig. 5 (d) is 244 a photograph of the electronic board.

245 A significant subset of the olive oil samples have been tested using both the bench-top instrument set-up described in Section 2.1 and the embedded system presented above to compare the results.

Fig. 6 (a) shows a plot of $\log _{10}\left(\mathrm{D}_{\text {OUT }} / 4096\right)$ vs. time (acquisitions every 5 seconds) for 3 samples featuring different values of total phenol content (the division by 4096 represents a normalization necessary for comparison with $\mathrm{V}_{\text {out }} / \mathrm{V}_{\mathrm{M}}$ of sub-Section 3.1). As can be seen, higher total phenol content results in lower values of measured $\mathrm{D}_{\text {OUT. }}$. Measurements on a set of 10 olive oil samples result in a linear correlation between $\log _{10}\left(\mathrm{D}_{\mathrm{OUT}} / 4096\right)$ and total phenol content $\left(\mathrm{Y}=-4.978 \cdot 10^{-4} \cdot \mathrm{X}\right.$ $\left.-4.7 \cdot 10^{-3}\right)$

In Fig. 6 (b) DouT/4096 is plotted vs. time for 3 samples featuring different values of peroxide value 2557 olive oil samples result in a linear correlation between $\mathrm{D}_{\text {OUT }} / 4096$ and the peroxide value $(\mathrm{Y}=$ $\left.8.532 \cdot 10^{-3} \cdot X+0.893\right)$

The linear regression equations have been used to compare the estimated values of both peroxide value and total phenol content with those obtained with the reference methods and the calculated 
259 determination coefficient $\mathrm{R}^{2}$ differs by less than $1.6 \%$ between the two measuring systems. In 260 particular, in the case of peroxide value, $\mathrm{R}^{2}=0.901$ and 0.887 for the bench-top set-up and the 261 embedded system, respectively; while with total phenol content $\mathrm{R}^{2}=0.932$ and 0.922 for the bench262 top set-up and the embedded system, respectively. The agreement between the two measuring 263 systems is very good.

\section{Conclusions}

266 A novel technique to measure peroxide value and total phenol content in olive oil has been 267 presented that is based on optical density measurements of a suitable reagent inoculated with the 268 olive oil of interest. The technique, suitable to be realized in the form of a low-cost, embedded 269 electronic system, has been tested using an experimental set-up built with bench-top 270 instrumentation and the results show that it can estimate with good accuracy the peroxide value $\left(\mathrm{R}^{2}\right.$ $271=0.883)$ and the total phenol content $\left(\mathrm{R}^{2}=0.895\right)$ of olive oil in less than 10 minutes.

272 The proposed technique has been implemented in an electronic board to realize a portable 273 embedded system; the results obtained with such an instrument have been compared with those 274 coming from the bench-top set-up and an excellent agreement has been found.

275 The portable embedded system could be applied for fast and in-situ olive oil quality control and to assess the shelf-life without the need of trained personnel. Furthermore, it could be useful for a first evaluation of the total phenol content of virgin olive oils in case of interest of a productor to report on the lable the oilive oil claim, according with the EU Regulation 432/2012, stating that "Olive oil polyphenols contribute to the protection of blood lipids from oxidative stress", but admitted only if 280 at least $5 \mathrm{mg}$ of hydroxytyrosol and its derivatives (e.g. oleuropein complex and tyrosol) are contained in $20 \mathrm{~g}$ of olive oil. 
286 This work has been financially supported by the CESAR Project, RIDIIT program, funded by the 287 Ministry of Economic Development (Italy).

\section{References}

Abuznait, A.H., Qosa, H., Busnena, B.A., El Sayed, K.A., \& Kaddoumi, A. (2013). Olive-oilderived oleocanthal enhances $\beta$-amyloid clearance as a potential neuroprotective mechanism against Alzheimer's disease: in vitro and in vivo studies. ACS Chemical Neuroscience, 4(6), 973982.

Adhoum, N., \& Monser, L. (2008). Electrochemical sensor for hydroperoxides determination based on Prussian blue film modified electrode. Sensors and Actuators B: Chemical, 133, 588-592.

Apak, R., Guclu, K., Demirata, B., Ozurek, M., Celik, S.E., Bektasoglu, B., Berker, K.I. \& Ozyurt, D. (2007). Comparative evaluation of various total antioxidant capacity assays to phenolic compounds with CUPRAC assay. Molecules, 12, 1496-1547.

Armenta, S., Garrigues, S., \& de la Guardia, M. (2007). Determination of edible oil parameters by near infrared spectrometry. Analytica Chimica Acta, 596, 330-337.

Boselli, E., Di Lecce, G., Strabbioli, R., Pieralisi, G., \& Frega, N.G. (2009). Are virgin olive oils obtained below $27{ }^{\circ} \mathrm{C}$ better than those produced at higher temperatures? Food Science and 
310 Bou, R., Codony, R., Tres, A., Decker, E.A., \& Guardiola F. (2008). Determination of 311 hydroperoxides in foods and biological samples by the ferrous oxidation-xylenol orange method: A 312 review of the factors that influence the method's performance. Analytical Biochemistry 377, 1-15.

314 Capannesi, C., Palchetti, I., Mascini, M., \& Parenti, A. (2000). Electrochemical sensor and 315 biosensor for polyphenols detection in olive oils. Food Chemistry, 71, 553-562.

317 Cataldo, A., Piuzzi, E., Cannazza, G., \& De Benedetto, E. (2012). Classification and adulteration 318 control of vegetable oils based on microwave reflectometry analysis. Journal of Food Engineering, $319112,338-345$.

321 Cerretani, L., Giuliani, A., Maggio, R.M., Bendini, A., Gallina Toschi, T., \& Cichelli, A. (2010). 322 Rapid FTIR determination of water, phenolics and antioxidant activity of olive oil. European Journal of Lipid Science, 112, 1150-1157.

Cheeseman, J.M. (2006). Hydrogen peroxide concentrations in leaves under natural conditions. Journal of Experimental Botany, 57(10), 2435-2444.

EC 1991 Commission Regulation (EEC) 2568/91 of July $11^{\text {th }} 1991$ on characteristics of olive oil and on the relevant methods of analysis. Official EC Journal, L248, 0001-0083.

EC 2012 Commission Regulation (EC) 432/2012 of May 16 th on establishing a list of permitted 320 health claims made on foods, other than those referring to the reduction of disease risk and to children's development and health, Official Journal of the European Communities. 2012, L 136. 
335 Fiori, F., Di Lecce, G., Boselli, E., Pieralisi, G., \& Frega, N.G. (2014). Effects of olive paste fast 336 preheating on the quality of extra virgin olive oil during storage. Food Science and Technology, 58 $337 \quad 511-518$

339 Grossi, M., Di Lecce, G., Gallina Toschi, T., \& Riccò, B. (2013). A novel electrochemical method 340 for olive oil acidity determination. Proceedings of the 5th IEEE International Workshop on 341 Advances in Sensors and Interfaces (IWASI), 162-167.

343 Gutierrez-Rosales, F., Rios, J.J., \& Gomez-Rey, M.L. (2003). Main polyphenols in the bitter taste 344 of virgin olive oil. Structural confirmation by on-line high-performance liquid chromatography 345 electrospray ionization mass spectrometry. Journal of Agricultural and Food Chemistry, 51(20), $346 \quad 6021-6025$.

IOC/T.20/Doc No 29 (2009). Official methods of analysis. Determination of biophenols in olive oils by HPLC. International Olive Council - COI, Madrid.

Jiang, Z.Y., Woollard, A.C.S., \& Wolff, S.P. (1990). Hydrogen peroxide production during experimental protein glycation. FEBS Letters, 268(1), 69-71.

Kardash-Strochkova, E., Tur'yan, Y.I., \& Kuselman, I. (2001). Redox-potentiometric determination of peroxide value in edible oils without titration. Talanta, 54, 411-416.

Keys, A., Mienotti, A., Karvonen, M.J., Aravanis, C., Blackburn, H., Buzina, R., Djordjevic, B.S., 
362 Lerma-Garcia M. J., Simò-Alfonso, E.F., Chiavaro, E., Bendini, A., Lercker. G., \& Cerretani, L. 363 (2009). Study of Chemical Changes Produced in Virgin Olive Oils with Different Phenolic 364 Contents during an Accelerated Storage Treatment. Journal Agricultural and Food Chemistry, 57, $365 \quad 7834-7840$.

Machowetz, A., Poulsen, H.E., Gruendel, S., Weimann, A., Fito, M., Marrugat, J., De La Torre, R., 368 Salonen, J.T., Nyyssonen, K., Mursu, J., Nascetti, S., Gaddi, A., Kiesewetter, H., Baumler, H., 369 Selmi, H., Kaikkonen, J., Zunft, H.J.F., Convas, M.I., \& Koebnick, C. (2007). Effect of olive oils on biomarkers of oxidative DNA stress in Northern and Southern Europeans. The FASEB Journal, 21(1), 45-52.

Monti, M.C., Margarucci, L., Tosco, A., Riccio, R., \& Casapullo, A. (2011). New insights on the interaction mechanism between tau protein and oleocanthal, an extra-virgin olive-oil bioactive component. Food \& Function, 2(7), 423-428.

Notarnicola, M., Pisanti, S., Tutino, V., Bocale, D., Rotelli, M.T., Gentile, A., Memeo, V., Bifulco, M., Perri, E., \& Caruso, M.G (2011). Effects of olive oil polyphenols on fatty acid synthase gene expression and activity in human colorectal cancer cells. Genes \& Nutrition, 6(1), 63-69.

Ozdemir, D., \& Ozturk, B. (2007). Near infrared spectroscopic determination of olive oil adulteration with sunflower and corn oil. Journal of Food and Drug Analysis, 15, 40-47.

Ragni, L., Berardinelli, A., Cevoli, C., \& Valli, E. (2012). Assessment of the water content in extra virgin olive oils by Time Domain Reflectometry (TDR) and Partial Least Squares (PLS) regression methods. Journal of Food Engineering, 111, 66-72. 
388 Rodriguez-Mendez, M.L., Apetrei, C., \& de Saja, J.A. (2008). Evaluation of the polyphenolic 389 contento f extra virgin olive oils using an array of voltammetric sensors. Electrochimica Acta, 53, $390 \quad 5867-5872$.

Rotondi, A., Bendini, A., Cerretani, L., Mari, M., Lercker, G., \& Gallina Toschi, T. (2004). Effect of olive ripening degree on the oxidative stability and organoleptic properties of cv. Nostrana di Brisighella extra virgin olive oil. Journal of Agricultural and Food Chemistry, 52, 3649-3654.

Singleton, V.L., \& Rossi, J.A. (1965). Colorimetry of total phenolics with phosphomolybdicphosphotungstic acid reagents. American Journal of Enology and Viticulture, 16, 144-158.

Tasioula-Margari, M., \& Okogeri, O. (2001). Isolation and characterization of virgin olive oil phenolic compounds by HPLC/UV and GC-MS. Journal of Food Science, 66(4), 530-534.

Tulipani, S., Martinez Huelamo, M., Rotches Ribalta, M., Estruch, R., Escribano Ferrer, E., 403 Andres-Lacueva, C., Illan, M., \& Lamuela-Raventòs, R.M. (2012). Oil matrix effects on plasma 404 exposure and urinary excretion of phenolic compounds from tomato sauces: evidence from a human pilot study. Food Chemistry, 130, 581-590.

Zanoni, B. (2014). Which processing markers are recommended for measuring and monitoring the 408 transformation pathways of main components of olive oil ? Italian Journal of Food Science, 26, 1409 9. 

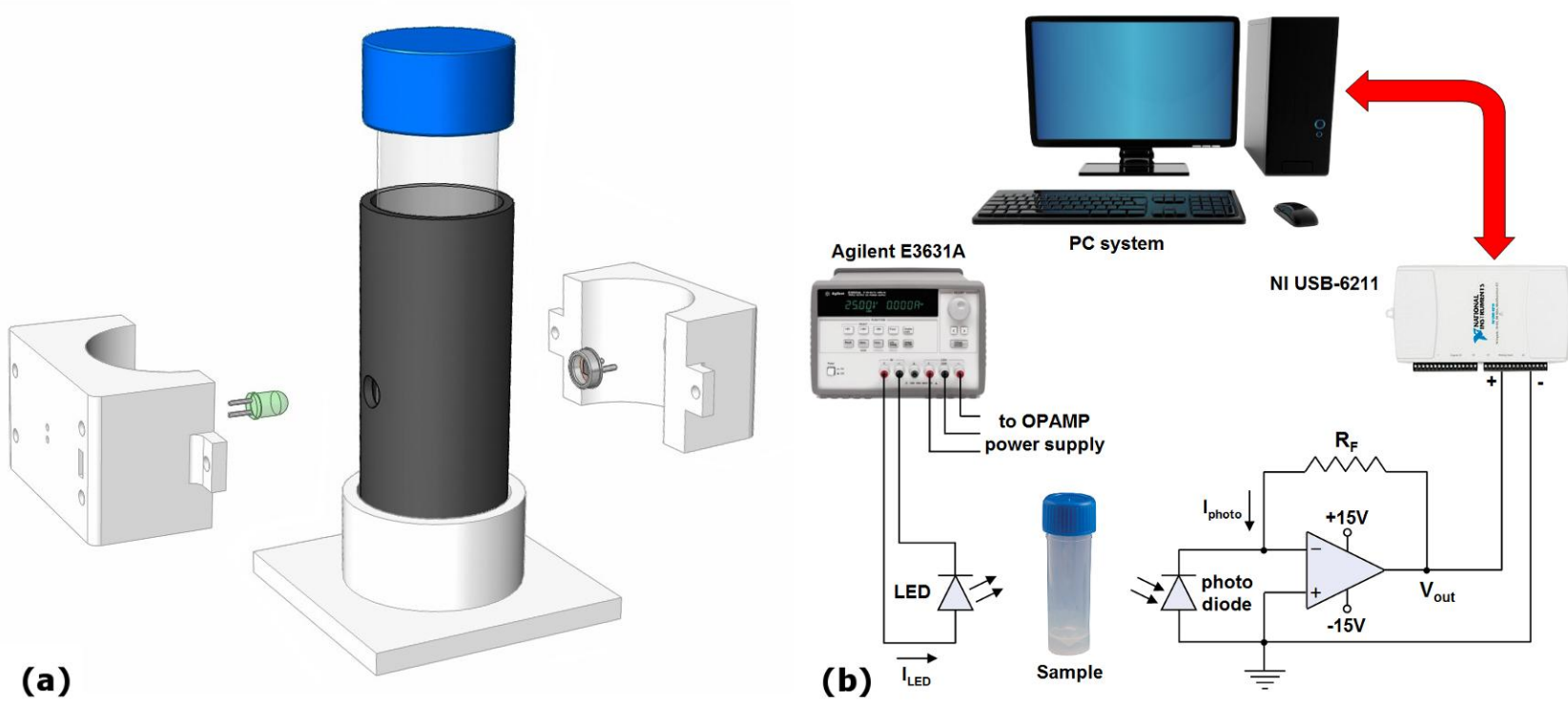

Fig. 1. The sensor used for the experiments of the present work (a) and the experimental set-up built 422 with bench-top instrumentation used for the measurements (b). 

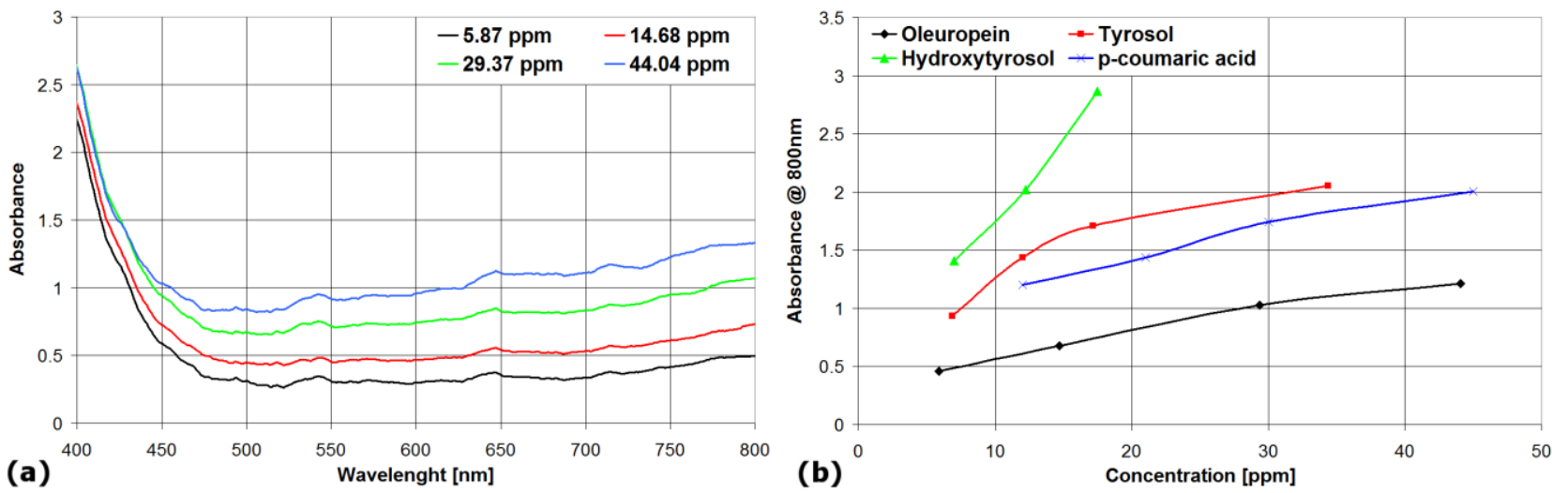

440 Fig. 2. Measured absorbance vs. wavelength in the range $400-800 \mathrm{~nm}$ for different concentrations 441 of oleuropein (a) and measured absorbance at $800 \mathrm{~nm}$ plotted vs. phenol content for different 442 phenolic compounds (b). 

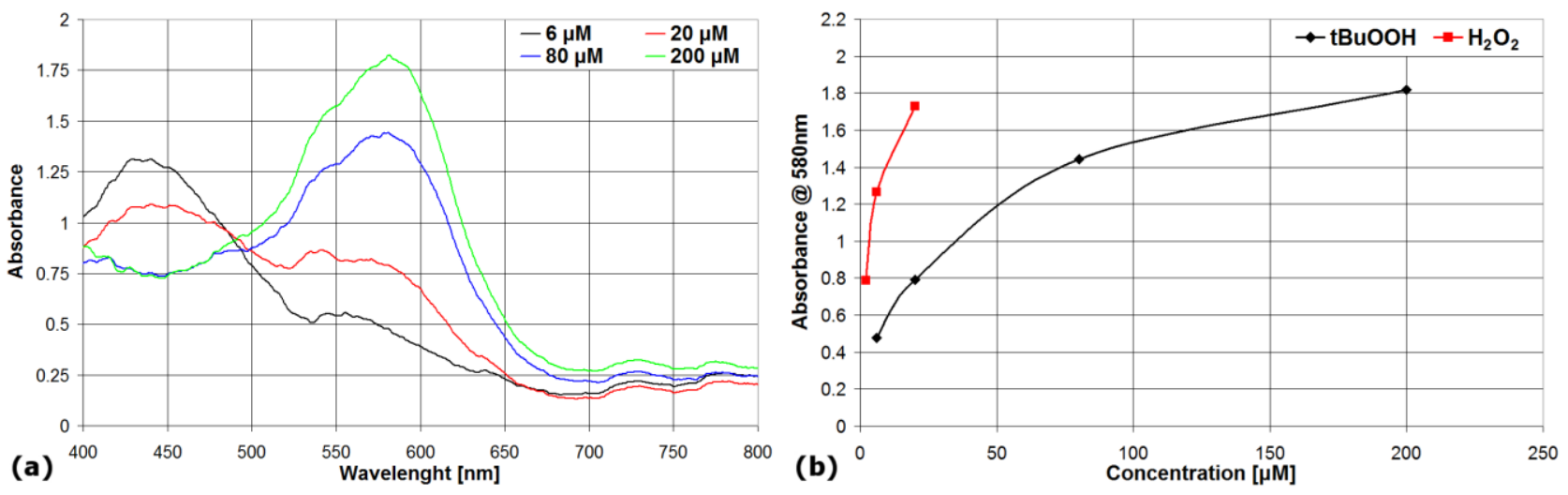

Fig. 3. Absorbance plotted vs. wavelength in the range $400-800 \mathrm{~nm}$ for different concentrations of 461 tert-butyl hydroperoxide (a) and measured absorbance at $580 \mathrm{~nm}$ plotted vs. concentration for tert462 butyl hydroperoxide as well as vs. hydrogen peroxide (b). 

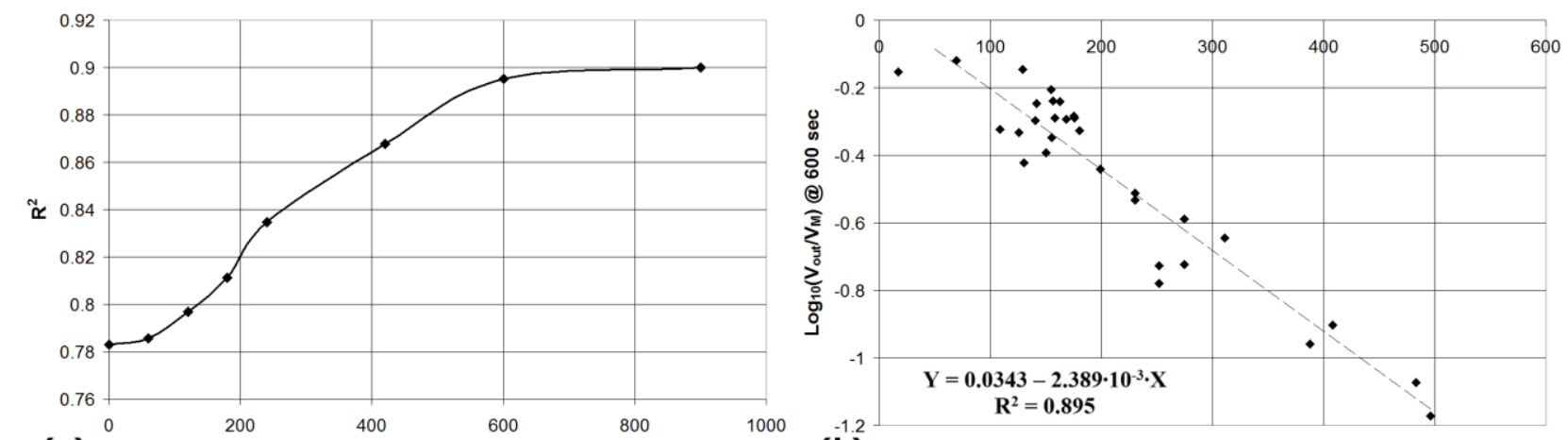

(a)

Time [seconds]

Total phenol content [ppm]
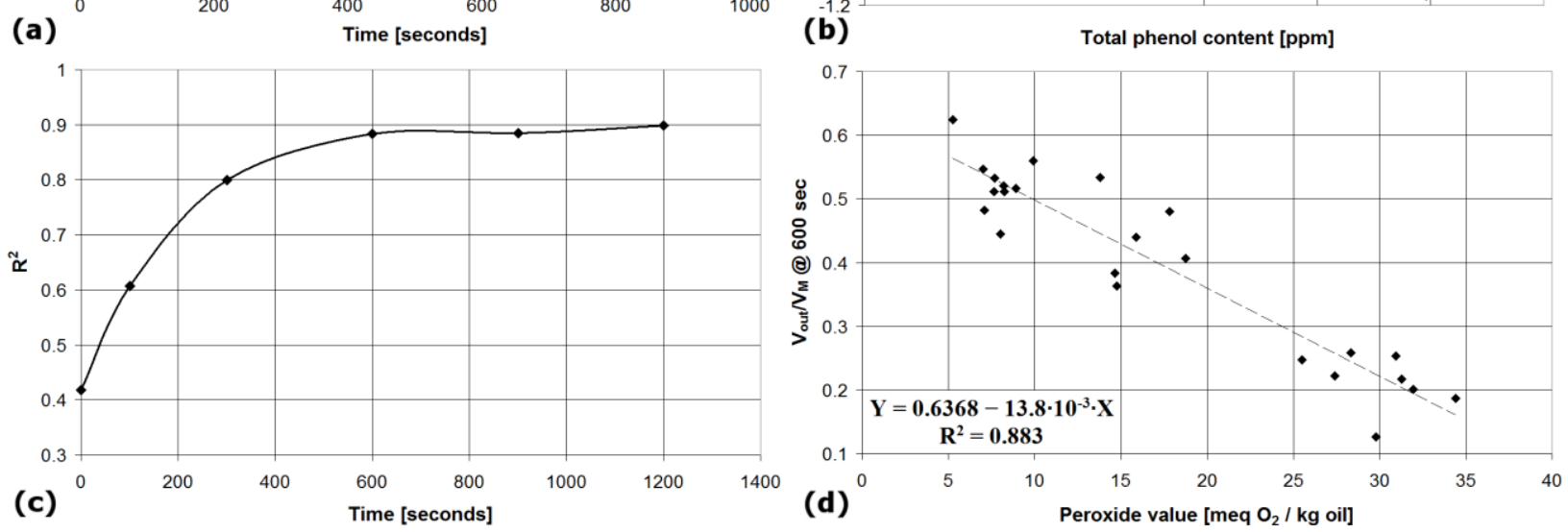

Fig. 4. Coefficient of determination $\mathrm{R}^{2}$ plotted vs. time for the total phenol content (a) and peroxide value (c). Scatter plot and linear regression line calculated for 600 seconds for total phenol content (b) and peroxide value (d). 

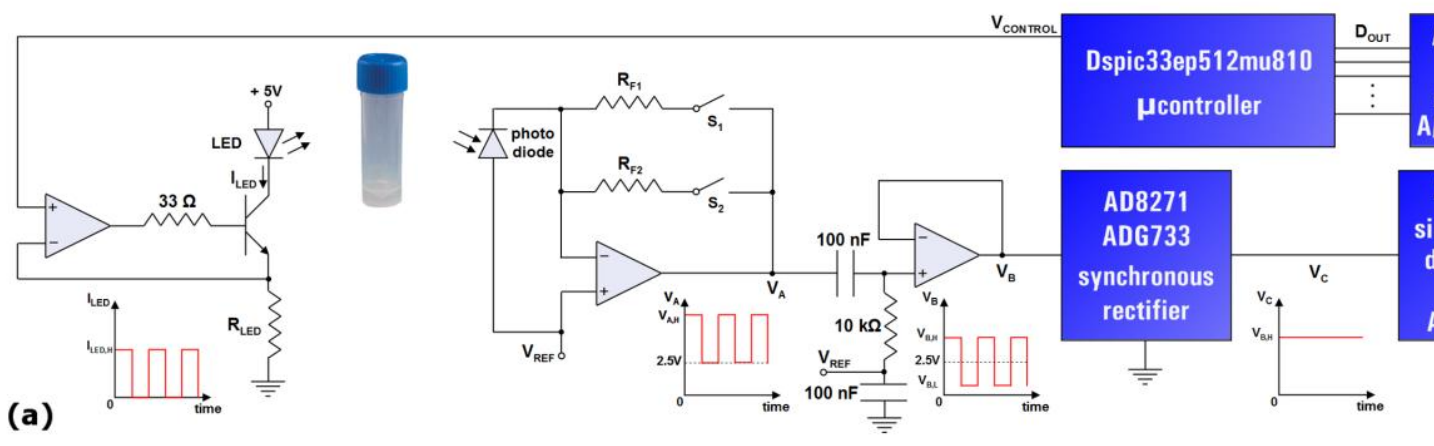

(a)
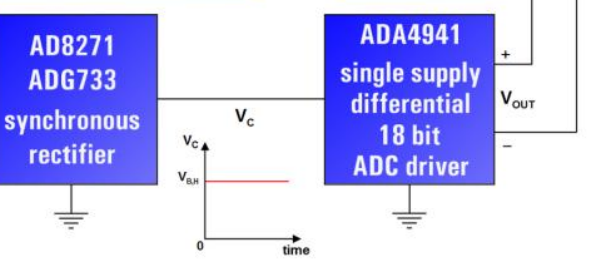

(b)
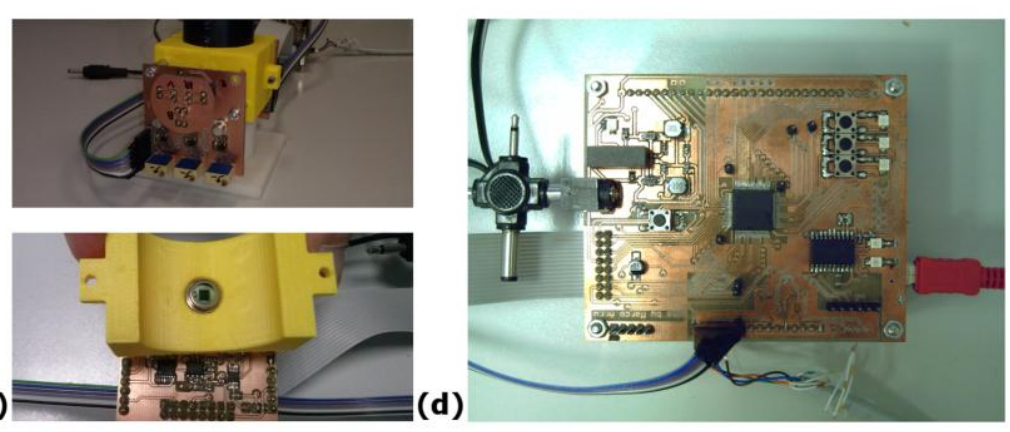

Fig. 5. Schematic of the electronic board of the embedded system realized in the present work (a); pictures of the sensor (b) and (c); photograph of the electronic board (d). 

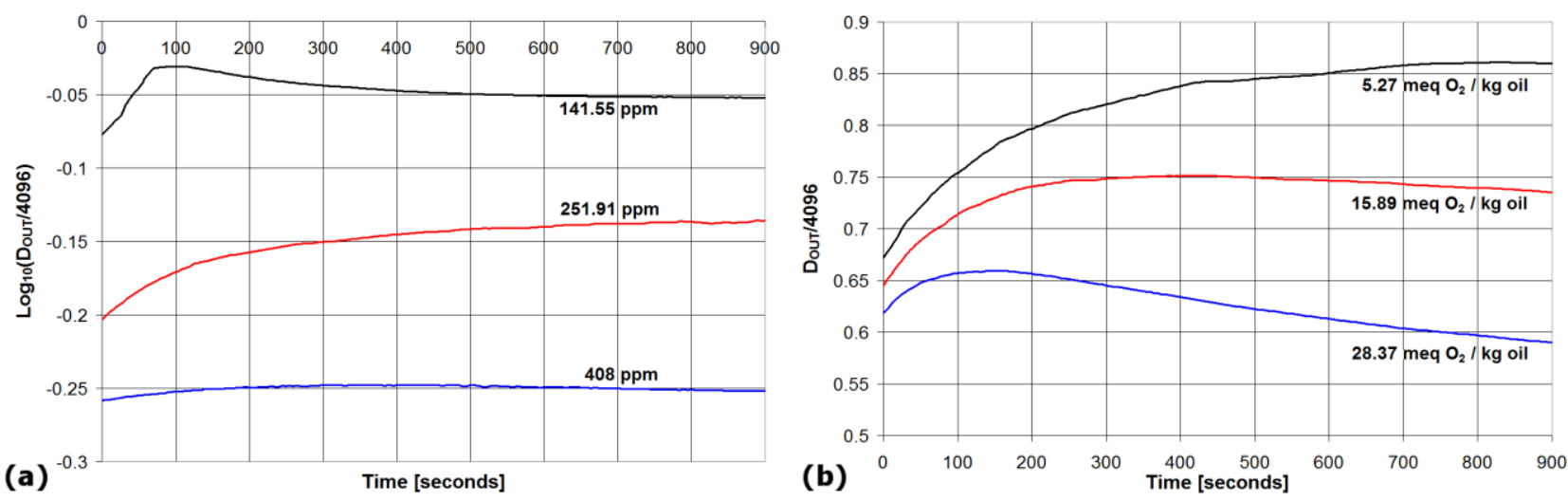

512 Fig. 6. Data measured with the designed electronic board for different oil samples vs. time in the 513 case of total phenol content (a) and peroxide value (b). 